\title{
PSMA7 Directly Interacts with NOD1 and Regulates its Function
}

\author{
Liuzhong Yang ${ }^{a}$ Zheng Tang ${ }^{b}$ Huiqiang Zhang ${ }^{c}$ Weizheng Kou ${ }^{a}$ Zhihong Lu $^{a}$ \\ Xiaorui Lia Qiuping Lid ${ }^{\mathrm{a}}$ Zhanhui Miao \\ aCancer Department of First Affiliated Hospital of Xinxiang Medical College Xinxiang, Henna, China; \\ 'Department of Forensic Pathology, Xinxiang Medical University, Xinxiang Henna, China; 'Tuberculosis \\ Department of First Affiliated Hospital of Xinxiang Medical College Xinxiang, Henna, China; 'Internal \\ medicine-cardiovascular Department of First Affiliated Hospital of Xinxiang Medical College Xinxiang, \\ Henna, China
}

\section{Key Words}

PSMA7 • NOD1 • Protein and protein interaction • Negative regulator

\begin{abstract}
Background/Aims: Recent reports showed that proteasome subunit alpha type-7 (PSMA7) was overexpressed in colorectal cancer. To investigate the mechanism of PSMA7 in promotion of colorectal cancer, we screened for its interaction partners. Methods and Results: This study found that PSMA7 associated with nucleotide-binding oligomerization domain-containing protein 1 (NOD1) by yeast two-hybrid screening, co-immunoprecipitation (IP), and GSTpull down assay. As shown by Western blotting and ubiquitin assay, PSMA7 downregulated the expression of NOD1 in a proteasome-dependent manner. Overexpression of PSMA7 in HCT116 cells resulted in an inhibition of NOD1-mediated apoptosis and NF- $\mathrm{KB}$ activation, whereas knockdown of PSMA7 by RNA interference enhanced NOD1 activity. Conclusion: Our data suggest that PSMA7 is a negative regulator of the NOD1, and may promote tumor growth by its inhibitory role on NOD1.
\end{abstract}

Copyright $(2013$ S. Karger AG, Basel

\section{Introduction}

Colorectal cancer is the third most common malignant neoplasm and the second leading cause of cancer deaths worldwide. It is widely accepted that the activation of oncogene k-ras and the inactivation of tumor suppressor genes p53, DCC/SMAD4, and APC, contribute to the 
progression and metastasis of colorectal cancer [1-4]. However, the molecular pathogenesis of colorectal cancer remains poorly understood. Recent studies suggested that PSMA7, an alpha-type subunit of the proteasome, is overexpressed in colorectal cancer and associated with its hepatic metastasis [5-7]. The proteasome is a multicatalytic proteinase complex with a highly ordered structure composed of 2 complexes, a $20 \mathrm{~S}$ core and a $19 \mathrm{~S}$ regulator [8]. The $19 \mathrm{~S}$ complex is required for the recognition of poly-ubiquitinated protein substrates that are degraded inside the 20S core complex. PSMA7 is a subunit of proteasome $20 \mathrm{~S}$ core complex. The role of PSMA7 in proteasome is well defined; however its function in tumorigenesis has not been fully elucidated.

NOD1 is a cytoplasmic protein that belongs to Nod-like receptor (NLR) family [9]. The primary function of this gene is thought to be a sensor of specific bacterial molecules, $\gamma$-Dglutamyl-meso-diaminopilemic acids (iE-DAP), which are derived from the bacterial cell wall peptidoglycan. NOD1 binding to its ligands triggers the secretion of pro-inflammatory cytokines through the activation of both NF- $\kappa$ B and MAPK pathways. Nod1 deficient mice or cell lines are shown to be more susceptible to certain pathogenic microbes, such as Shigella flexneri, Helicobacter pylori, and Listeria monocytogenes [10-12]. Recent reports have shown that Nod1 controlled tumor cell growth beyond its role in responses to infection. Nod1 has been shown to increase apoptosis, and overexpression of Nod1 resulted in an activation of caspase-8-promoted cell death [13]. In breast cancer, the absence of Nod1 was associated with tumor growth and an increased sensitivity to estrogen stimulation [14]. It was also reported that Nod1 protected the intestine from inflammation-induced tumorigenesis [15].

In this study, we demonstrate that PSMA7 physically interacts with NOD1 and decreases the expression of NOD1, providing a novel mechanism for PSMA7 promotes tumorigenesis.

\section{Materials and Methods}

\section{Cell culture and tansfection}

Human embryonic kidney cell line 293 and colon carcinoma cell line HCT116 were grown in DMEM supplemented with fetal bovine serum (FBS, Hyclone) and maintained at $37{ }^{\circ} \mathrm{C}$ under $5 \% \mathrm{CO}_{2}$ in a humidified incubator. For transfections, cells were plated to 85-90\% confluency and transfected with Lipofectamine 2000 (Invitrogen). For transient knockdown of PSMA7, PSMA7 siRNA oligonucleotide (Santa Cruz Biotechnology) was transfected following the manufacturer's instructions.

\section{Luciferase reporter assays}

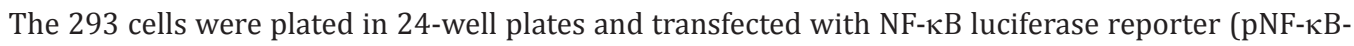
luc, $100 \mathrm{ng}$ ) and pRL-TK (2 ng) together with $100 \mathrm{ng}$ plasmid encoding NOD1 and increasing amounts of Myc-PSMA7 plasmid. After $24 \mathrm{~h}$, transfected cells were collected, and luciferase activity was measured using the dual luciferase reporter assay kit (Promega Corp.). All experiments were repeated at least three times.

\section{IP and immunoblot analysis}

Cell extracts were prepared in NP-40 lysis buffer containing $50 \mathrm{mM}$ Tris- $\mathrm{HCl}$ (pH 8.0), $150 \mathrm{mM} \mathrm{NaCl}$, and $1 \%$ NP-40 in the presence of Complete ${ }^{\circledR}$ protease inhibitor cocktail (Roche Applied Science). Supernatants of cell lysates were subjected to IP with anti-Flag (Sigma-Aldrich). For endogenous IP, anti-NOD1 antibody and protein A/G Sepharose beads were added into the lysates instead. The immunoprecipitated proteins were blotted onto PVDF membrane after separated on 8-12\% SDS-PAGE, and detected with anti-Flag, antiMyc (Sigma-Aldrich), anti-PSMA7 (Proteintech), anti-caspase 8, anti-NOD1 (Santa Cruz Biotechnology), or anti- $\beta$-tubulin (Sigma-Aldrich) antibody.

\section{GST-pull down and far-Western}

For GST-pull down experiments, cell lysates containing Flag-NOD1 were incubated for $2 \mathrm{~h}$ at $4{ }^{\circ} \mathrm{C}$ with $1 \mu \mathrm{g}$ purified GST or GST-PSMA7 fusion proteins bound to glutathione beads. The immunoprecipitates were washed with NP-40 lysis buffer and then subjected to immunoblotting analysis. For far-Western assay, 
Fig. 1. PSMA7 associates with NOD1. a: The 293 cells were co-transfected with Flag-NOD1or Flag-vector together with Myc-PSMA7. After $24 \mathrm{~h}$ of transfection, coIP experiment was performed by anti-Flag agarose. The interaction was detected by immunoblotting with Flag and Myc antibody. b: Lysates from HCT116 cells was immunoprecipitated using NOD1 specific antibody and control normal IgG. The immuno-complex was separated in SDSPAGE and analyzed by immunoblotting with anti-NOD1 and PSMA7 antibodies. c: The 293 cells transfected with Flag-NOD1 expression plasmid were lysed by NP-40 lysis buffer. The GST or GST-PSMA7 fusion protein absorbates from cell lysates were analyzed by immunoblotting with antiFlag antibody (top). Loading of the GST proteins was assessed by immunoblotting with anti-GST antibody (bottom). d: AntiFlag immunoprecipitates from cells trans-

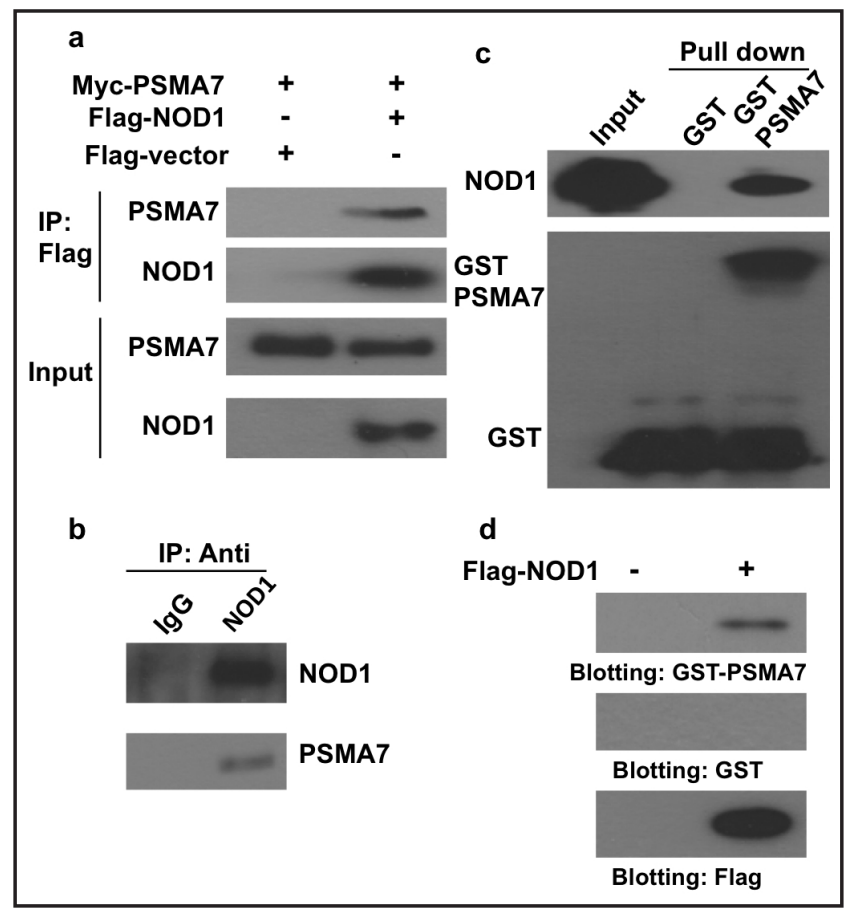

fected with Flag-NOD1 were blotted onto NC membrane, which was then incubated with soluble GST-PSMA7 and GST for $2 \mathrm{~h}$ and analyzed with anti-GST (top, middle) or anti-Flag (bottom) antibody.

Flag-NOD1 purified from 293 cells was separated by SDS-PAGE and then blotted onto nitrocellulose (NC) membranes, which were subsequently incubated with purified GST or GST-fusion proteins for $1 \mathrm{~h}$ at room temperature. The GST fusion proteins binding to NC were probed with anti-GST antibody.

In vivo ubiquitination assays

Cells were co-transfected with Flag-NOD1, HA-ubiquitin, and different doses of Myc-PSMA7. After $24 \mathrm{~h}$, cells were grown in medium containing MG132 $(20 \mu \mathrm{M})$ for $8 \mathrm{~h}$. Immunoprecipitates with anti-Flag agarose were analyzed by immunoblotting with anti-HA antibody. Flag-NOD1 was used as equal loading control.

\section{Analysis of apoptosis by flow cytometry}

Annexin V Staining Kit was purchased from BD Pharmingen (San Jose, CA, USA). After transfection with expression plasmid or siRNA for $48 \mathrm{~h}$, the cells were digested with trypsin to make a single-cell suspension, washed 3 times with PBS, resuspended in binding buffer, and adjusted to a density of $1 \times 10^{6}$ cells $/ \mathrm{ml}$. In each tube, $100 \mu \mathrm{l}$ of cell suspension were added with $15 \mu \mathrm{l}$ of FITC-conjugated Annexin V, incubated at $4{ }^{\circ} \mathrm{C}$ for $20 \mathrm{~min}$, and analyzed then for cell apoptosis by flow cytometry.

\section{Results}

PSMA7 interacts with NOD1 in vivo and in vitro

To study the mechanism of tumorigenesis induced by PSMA7 in colorectal cancer, interaction partners of PSMA7 were screened by yeast two-hybrid assay. PSMA7 was cloned into pGBKT7 vector and used as bait. From $7 \times 10^{6}$ transfectants, NOD1 was identified (data not shown). To demonstrate the results of yeast two-hybrid screening, 293 cells were cotransfected with Flag-tagged NOD1 and Myc-tagged PSMA7. Following transfection, co-IP experiment was performed by anti-Flag agrose. As expected, PSMA7 was detected in the 
Fig. 2. PSMA7 promotes NOD1 degradation. a: HCT116 cells were transfected with NOD1 and increasing amount of PSMA7 expression plasmids $(0,0.5$, $1,1.5$, and $2 \mu \mathrm{g}$ ). The lysates were separated by SDS-PAGE, and then blotted with Flag and Myc antibodies respectively. $\beta$-Tubulin was used as equal loading control. b: HCT116 cells were transfected with increasing amount of Myc-PSMA7 expressing plasmids $(0,1$, 1.5 , and $2 \mu \mathrm{g}$ ). Total RNA was extracted, and relative mRNA levels of NOD1 were monitored by semi-quantitative RTPCR. c: HCT116 cells were transfected with Myc-PSMA7 expressing plasmids, the expression of endogenous NOD1 was detected by NOD1 specific antibody. d: HCT116 cells were co-transfected

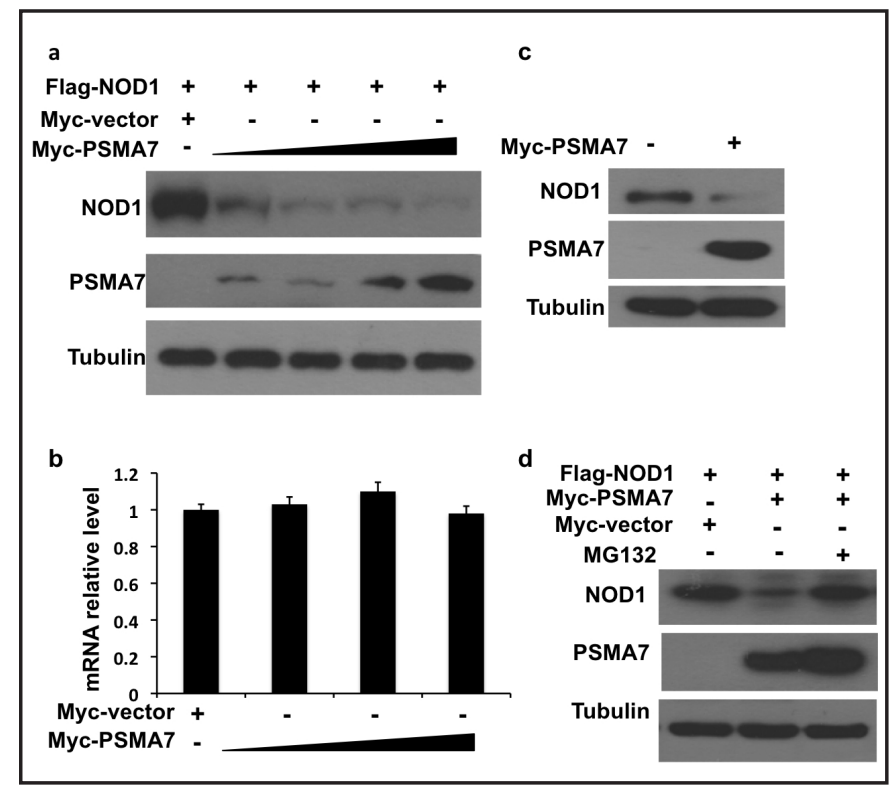
with NOD1 and PSMA7 expression plasmids. After 24 h, MG132 or vehicle control was added to the culture medium. The expression of NOD1 was detected by Flag antibody.

IP complex with NOD1, but not with a negative control (Fig. 1a). Then, the specificity of the interaction between endogenous NOD1 and PSMA7 was also examined by co-IP analysis using NOD1 specific antibody and control normal IgG, which demonstrated that NOD1 was associated with endogenous PSMA7 in HCT116 cells (Fig. 1b). To show the interaction of PSMA7 and MAVS in vitro, cell lysates from 293 cells expressing Flag-NOD1 were incubated with GST or GST-PSMA7 fusion protein. As shown in Fig. 1c, MAVS was associated with GST-PSMA7, but not GST. To determine the direct binding between these two proteins, immunoprecipitates prepared from cells expressing Flag-NOD1 were subjected to SDS-PAGE and then blotted onto NC membrane. After incubation with purified soluble GST-PSMA7, the NC membrane was immunoblotted with an HRP-conjugated anti-GST antibody. The results showed that NOD1 bound to PSMA7 directly (Fig. 1d).

\section{PSMA7 downregulates the expression NOD1}

Nod1 plays a crucial role in preventing tumorigenesis by protecting the intestinal epithelial barrier against injury and bacterial translocation. We detected the effect of PSMA7 on the expression of NOD1. Increasing PSMA7 expression plasmids were co-transfected with the same amounts of NOD1 into HCT116 cells, a significant reduction of NOD1 was found with PSMA7 transfection in a dose dependent manner (Fig. 2a). Semi-quantitative PCR showed that NOD1 mRNA level was not affected by PSMA7 overexpression (Fig. 2b). Endogenous NOD1 was also downregulated by PSMA7 plasmid transfection (Fig. 2c). To confirm that the degradation is mediated by proteasome, HCT116 cells were transfected with PSMA7 and treated with proteasome inhibitor MG132 or DMSO (control). Fig. $2 \mathrm{~d}$ shows that MG132 was able to reverse the reduction of NOD1, indicating that proteasome pathway is involved in PSMA7 mediated NOD1 instability.

\section{PSMA7 blocks NOD1-mediated apoptosis and NF- $\kappa B$ activation}

Activation of Nod1 initiates a variety of cellular responses including NF- $\kappa B$ activation and apoptosis. To assess the functional effect of PSMA7 on the reduction of NOD1, apoptosis induced by NOD1 was detected. With the addition of PSMA7, apoptosis caused by the activation of NOD1 was decreased (Fig. 3a). The cleavage of pro-caspase 8 produces the active form of caspase-8, which is a critical step in apoptosis event, and several lines of evidence 
Fig. 3. PSMA7 impairs NOD1mediated apoptosis and NF-kB activation. a: HCT116 cells were co-transfected with NOD1 and PSMA7, and the cells were trypsinized after $48 \mathrm{~h}$. The cells were stained using FITC-Annexin V, and then analyzed by flow cytometry to show cell apoptosis. b: NOD1 and PSMA7 or control plasmids were co-transfected into HCT116 cells. The cleavage of pro-caspase 8 was detected by immunoblotting. c: The 293 cells were transfected with NOD1, increasing amounts of PSMA7 plasmid, together with NF- $\kappa \mathrm{B}$ luciferase and pRL plasmid. The luciferase activity was measured $24 \mathrm{~h}$ later and normalized to transfection efficiency. The error bar represents SD from the mean value of triplicate experiments. d: HCT116 cells

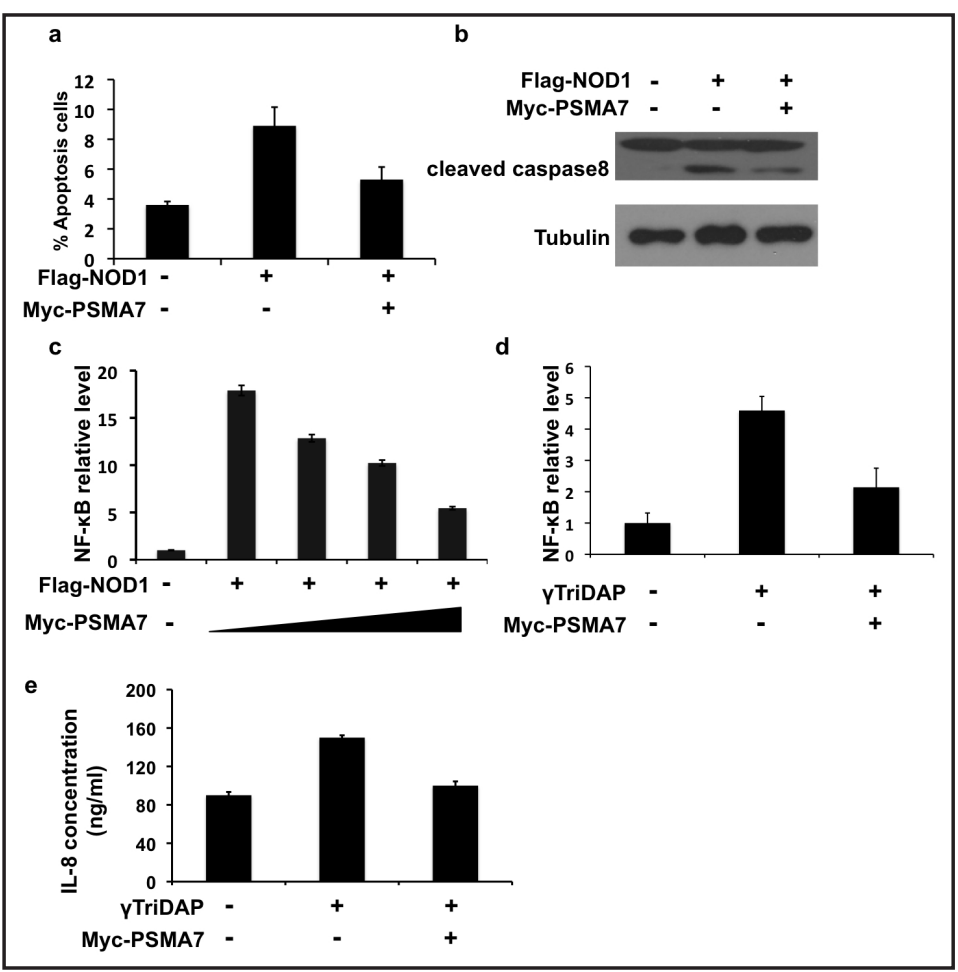
were transfected with increasing amounts of PSMA7 plasmid, NF- $\kappa B$ luciferase and pRL plasmids, and then stimulated with $\gamma$ TriDAP for $24 \mathrm{~h}$. NF- $\mathrm{KB}$ activation in response to NOD1 ligands in the absence or presence of PSMA7 was measured by luciferase activity assay. e: HCT116 cells were transfected with PSMA7 and then stimulated with $\gamma$ TriDAP for $24 \mathrm{~h}$, the amount of IL-8 from the supernatants was mesueaed by ELISA.

Fig. 4. Knockdown of PSMA7 promotes NOD1-mediated apoptosis and NF- $\kappa$ B activation. a: HCT116 cells were transfected with siPSMA7 or control siRNA. After $72 \mathrm{~h}$, whole cell lysates were analyzed by immunoblotting with NOD1, PSMA7, and $\beta$-Tubulin antibody. b: HCT116 cells were transfected with SiPSMA7 or control siRNA together with NF- $\kappa$ B luciferase and pRL plasmid. After treated with $\gamma$ TriDAP, the luciferase activity was measured and normalized to transfection efficiency. c: HCT116 cells were treated with siPSMA7 or control siRNA for $48 \mathrm{~h}$, and then the cells were treated by $\gamma$ TriDAP for $24 \mathrm{~h}$. Apoptosis was analyzed by FITC-Annexin $\mathrm{V}$ staining and

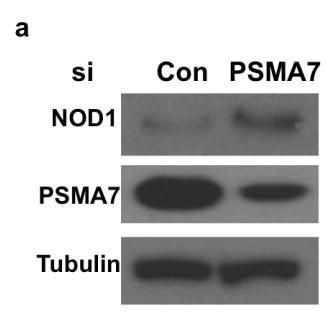

c

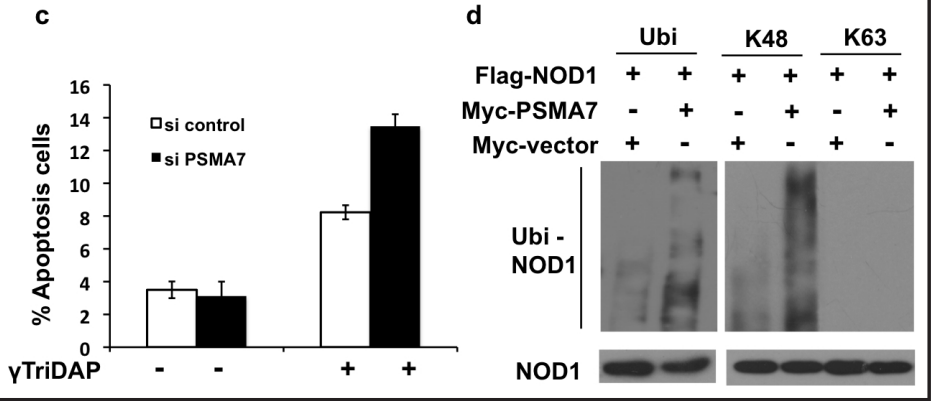
flow cytometry assay. $\mathrm{d}$ : The 293 cells were transfected with increasing amounts of PSMA7, Flag-NOD1, and HA-ubiquitin or HA-ubiquitin (k48, k63) plasmids. After $24 \mathrm{~h}$, cells were added with MG132 for an additional 6 h. Anti-Flag immunoprecipitates were analyzed by immunoblotting with HA and Flag antibody.

showed that NOD1-dependent apoptosis is a caspase 8-mediated event [10]. Caspase 8 was cleaved in the NOD1 overexpressing cells; however, this cleavage was partially decreased by 
PSMA7 (Fig. 3b). The effect of PSMA7 on NF-kB activity induced by NOD1 was also analyzed. The 293 cells were transfected with NF-кB luciferase reporter plus equal amount of NOD1 and increasing amounts of PSMA7 plasmids. As shown in Fig. 3c, NOD1 overexpression in 293 cells activated the NF- $\mathrm{BB}$ promoter, whereas such activation was inhibited by PSMA7 in a dose-dependent manner. Addition of $\gamma$ TriDAP to HCT116 cells induced increasing NF- $\kappa B$ promoter activity, and this activation was inhibited by PSMA7 too (Fig. 3d). We detected the role of PSMA7 on NF- $\kappa$ B associated gene (IL-8) stimulated by $\gamma$ TriDAP. From Fig. 3e, we can see that PSMA7 also downregulated IL-8 expression by ELISA.

\section{Depletion of PSMA7 increases NOD1 expression and activity}

As shown in Fig. 2, NOD1 was downregulated by PSMA7 plasmid transfection; consistently, specific knockdown of PSMA7 expression by RNA interference increased endogenous NOD1 abundance as compared to control siRNA in HCT116 cells (Fig. 4a). $\gamma$ TriDAP is a natural PAMP (pathogen-associated molecular pattern) of NOD1. In this experiment, we used the $\gamma$ TriDAP to activate the endogenous NOD1 signal pathway in HCT116 cells. Transfection of PSMA7-specific siRNA, but not control siRNA enhanced NF-кB luciferase activity induced by NOD1 activation, in coincidence with the inhibitory effects of PSMA7 in overexpression experiments (Fig. 4b). The siPSMA7 also increased the apoptosis induced by $\gamma$ TriDAP treatment (Fig. 4c).

\section{PSMA7 increases ubiquitination of NOD1}

To further explore the mechanism by which PSMA7 mediated NOD1 degradation, 293 cells were transfected with plasmids expressing Flag-NOD1, Myc-PSMA7, and HA-ubiquitin. Twenty hours after transfection, MG132 was added to a final concentration of $20 \mathrm{nM}$ for $12 \mathrm{~h}$. As shown in Fig. 4d, PSMA7 led to an increased ubiquitination of NOD1. We used Lys48 (K48) and Lys63 (K63) only ubiquitin expression plasmid to find out the form of ubiquitination linked. From Fig. 4d, we confirmed that PSMA7 promoted K48-linked poly-ubiquitination status of NOD1, but not K63-linked form.

\section{Discussion}

Tumorigenesis is a complex process in which normal cells experience multiple genetic and epigenetic alterations to transform into malignant tumor cells. Recent findings indicated the relationship between PSMA7 expression and the clinicopathological features of colorectal cancer and suggested that PSMA7 may be molecular targets for colorectal cancer therapy $[5,6,16]$. However, it is unknown how PSMA7 critically regulates the tumorigensis of colorectal cancer cells. In this report, several lines of evidence supported the notion that PSMA7 functions partially through downregulation NOD1: 1) PSMA7 interacts with NOD1 as determined by GST pull down, co-IP, and far-Western; 2) PSMA7 increases NOD1 degradation by promoting its ubiquitination; 3) PSMA7 downregulates NOD1 expression and NOD1mediated apoptosis and NF- $\mathrm{BB}$ activity; and 4) knockdown of PSMA7 partially rescued the expression and activity of NOD1.

As a component of proteasome, PSMA7 interacts with certain proteins and targets them for proteasome-dependent degradation. Cho et al. showed that PSMA7 bind with hypoxiainducible factor- $1 \alpha$ and promote its degradation through proteasome-dependent pathway [17]. HBVx (hepatitis B virus X protein) is a binding partner with PSMA7, which is degraded by the ubiquitin-proteasome system $[18,19]$. Jia et al. showed that PSMA7 impairs IFN- $\beta$ promoter activity by increasing ubiquitination of mitochondrial antiviral signaling (MAVS) during viral infection [20]. Because proteasome subunits can be exchanged with exogenous subunits in vivo and in vitro, PSMA7 may have a role in recruiting specific substrates to the proteasome complex through subunit exchanging and proteasome assembling [21]. The present study finds that NOD1 is a novel substrate of PSMA7 and also trapped by the latter in proteasome-dependent degradation, which can be blocked by the addition 
of proteasome inhibitor MG132. Sensing of commensal microorganisms by the innate immune system maintains intestinal homeostasis and induces healing responses following injury, which confers protection against colitis and colorectal cancer [22]. As an important pattern recognition sensor, NOD1 monitors damage by recognition of the derivatives of lipopolysaccharides, and thus protects host from colitis and inflammation-induced tumorigenesis. Recent study shows that NOD1 also promotes apoptosis [13, 14, 23], and defective apoptosis represents a major causative factor in the development and progression of cancer [24]. To better understand functional effects and mechanisms involving in the regulation of NOD1, a number of proteins that interact with NOD1 have been identified and characterized. Previous reports showed that while RIPK2, SGT1, and BID are positive regulators, CENTB1 is the only one that directly and negatively regulates NOD1 activity [25, 26]. CENTB1 downregulates NF- $\mathrm{kB}$ activation via NOD1 and NOD2 pathways; however, the role of CENTB1 in regulation of apoptosis induced by NOD1 was not detected. Here, we report a novel negative regulator of NOD1, PSMA7, which downregulates both the expression and activity of NOD1 and inhibits the apoptosis induced by NOD1.

In summary, we have identified a novel regulatory function of PSMA7 in colorectal cancer. By forming a complex with NOD1, PSMA7 promotes NOD1 degradative polyubiquitination, decreases its protein expression and apoptosis induced by NOD1 activation. Our results also suggest a possible mechanism of PSMA7 in promoting colorectal cancer by impairing the function NOD1 and intestinal homeostasis. Therefore, PSMA7 may form a potential target for the development of clinical therapy for tumors caused by NOD1 dysfunction.

\section{Conflict of Interest}

None.

\section{References}

1 Conlin A, Smith G, Carey FA, Wolf CR, Steele RJ: The prognostic significance of k-ras, p53, and apc mutations in colorectal carcinoma. Gut 2005;54:1283-1286.

2 Esteller M, Gonzalez S, Risques RA, Marcuello E, Mangues R, Germa JR, Herman JG, Capella G, Peinado MA: K-ras and p16 aberrations confer poor prognosis in human colorectal cancer. J Clin Oncol 2001;19:299304.

- 3 Poon RT, Ng IO, Lau C, Yu WC, Fan ST, Wong J: Correlation of serum basic fibroblast growth factor levels with clinicopathologic features and postoperative recurrence in hepatocellular carcinoma. Am J Surg 2001;182:298-304.

4 Xu Y, Pasche B: Tgf-beta signaling alterations and susceptibility to colorectal cancer. Hum Mol Genet 2007;16 Spec No 1:R14-20.

5 Hu XT, Chen W, Wang D, Shi QL, Zhang FB, Liao YQ, Jin M, He C: The proteasome subunit psma7 located on the 20q13 amplicon is overexpressed and associated with liver metastasis in colorectal cancer. Oncol Rep 2008;19:441-446.

6 Honma K, Takemasa I, Matoba R, Yamamoto Y, Takeshita F, Mori M, Monden M, Matsubara K, Ochiya T: Screening of potential molecular targets for colorectal cancer therapy. Int J Gen Med 2009;2:243-257.

-7 Hu XT, Chen W, Zhang FB, Shi QL, Hu JB, Geng SM, He C: Depletion of the proteasome subunit psma7 inhibits colorectal cancer cell tumorigenicity and migration. Oncol Rep 2009;22:1247-1252.

8 Murata S, Yashiroda H, Tanaka K: Molecular mechanisms of proteasome assembly. Nat Rev Mol Cell Biol 2009;10:104-115.

-9 Chamaillard M, Hashimoto M, Horie Y, Masumoto J, Qiu S, Saab L, Ogura Y, Kawasaki A, Fukase K, Kusumoto S, Valvano MA, Foster SJ, Mak TW, Nunez G, Inohara N: An essential role for nod1 in host recognition of bacterial peptidoglycan containing diaminopimelic acid. Nat Immunol 2003;4:702-707. 


\section{Cellular Physiology $\quad$ Cell Physiol Biochem 2013;31:952-959 and Biochemistry \\ Yang et al.: PSMA7 Downregulates the Activity of NOD1}

10 Girardin SE, Tournebize R, Mavris M, Page AL, Li X, Stark GR, Bertin J, DiStefano PS, Yaniv M, Sansonetti PJ, Philpott DJ: Card4/nod1 mediates nf-kappab and jnk activation by invasive shigella flexneri. EMBO Rep 2001;2:736-742.

11 Watanabe T, Asano N, Fichtner-Feigl S, Gorelick PL, Tsuji Y, Matsumoto Y, Chiba T, Fuss IJ, Kitani A, Strober W: Nod1 contributes to mouse host defense against helicobacter pylori via induction of type i ifn and activation of the isgf3 signaling pathway. J Clin Invest 2010;120:1645-1662.

12 Opitz B, Puschel A, Beermann W, Hocke AC, Forster S, Schmeck B, van Laak V, Chakraborty T, Suttorp N, Hippenstiel S: Listeria monocytogenes activated p38 mapk and induced il-8 secretion in a nucleotidebinding oligomerization domain 1-dependent manner in endothelial cells. J Immunol 2006;176:484-490.

-13 da Silva Correia J, Miranda Y, Leonard N, Hsu J, Ulevitch RJ: Regulation of nod1-mediated signaling pathways. Cell Death Differ 2007;14:830-839.

14 da Silva Correia J, Miranda Y, Austin-Brown N, Hsu J, Mathison J, Xiang R, Zhou H, Li Q, Han J, Ulevitch RJ: Nod1-dependent control of tumor growth. Proc Natl Acad Sci U S A 2006;103:1840-1845.

15 Chen GY, Shaw MH, Redondo G, Nunez G: The innate immune receptor nod1 protects the intestine from inflammation-induced tumorigenesis. Cancer Res 2008;68:10060-10067.

16 Smith L, Qutob O, Watson MB, Beavis AW, Potts D, Welham KJ, Garimella V, Lind MJ, Drew PJ, Cawkwell L: Proteomic identification of putative biomarkers of radiotherapy resistance: A possible role for the $26 \mathrm{~s}$ proteasome? Neoplasia 2009;11:1194-1207.

17 Cho S, Choi YJ, Kim JM, Jeong ST, Kim JH, Kim SH, Ryu SE: Binding and regulation of hif-1alpha by a subunit of the proteasome complex, psma7. FEBS Lett 2001;498:62-66.

18 Hu Z, Zhang Z, Doo E, Coux O, Goldberg AL, Liang TJ: Hepatitis b virus x protein is both a substrate and a potential inhibitor of the proteasome complex. J Virol 1999;73:7231-7240.

19 Huang J, Kwong J, Sun EC, Liang TJ: Proteasome complex as a potential cellular target of hepatitis b virus x protein. J Virol 1996;70:5582-5591.

-20 Jia Y, Song T, Wei C, Ni C, Zheng Z, Xu Q, Ma H, Li L, Zhang Y, He X, Xu Y, Shi W, Zhong H: Negative regulation of mavs-mediated innate immune response by psma7. J Immunol 2009;183:4241-4248.

21 Fruh K, Gossen M, Wang K, Bujard H, Peterson PA, Yang Y: Displacement of housekeeping proteasome subunits by mhc-encoded lmps: A newly discovered mechanism for modulating the multicatalytic proteinase complex. EMBO J 1994;13:3236-3244.

22 Saleh M, Trinchieri G: Innate immune mechanisms of colitis and colitis-associated colorectal cancer. Nat Rev Immunol 2011;11:9-20.

-23 Bertin J, Nir WJ, Fischer CM, Tayber OV, Errada PR, Grant JR, Keilty JJ, Gosselin ML, Robison KE, Wong GH, Glucksmann MA, DiStefano PS: Human card4 protein is a novel ced-4/apaf-1 cell death family member that activates nf-kappab. J Biol Chem 1999;274:12955-12958.

24 Kasibhatla S, Tseng B: Why target apoptosis in cancer treatment? Mol Cancer Ther 2003;2:573-580.

25 Inohara N, Koseki T, Lin J, del Peso L, Lucas PC, Chen FF, Ogura Y, Nunez G: An induced proximity model for nf-kappa b activation in the nod1/rick and rip signaling pathways. J Biol Chem 2000;275:27823-27831.

-26 Inohara N, Ogura Y, Chen FF, Muto A, Nunez G: Human nod1 confers responsiveness to bacterial lipopolysaccharides. J Biol Chem 2001;276:2551-2554. 